\title{
La dévotion aux saintes dans les Miracles de Nostre Dame de Gautier de Coinci : une érotique du langage aux frontières de l'interdit
}

\section{Claire Donnat-Aracil}

\section{OpenEdition}

\section{Journals}

Édition électronique

URL : http://journals.openedition.org/questes/4456

DOI : 10.4000/questes.4456

ISSN : 2109-9472

Éditeur

Les Amis de Questes

\section{Édition imprimée}

Date de publication : 31 janvier 2018

Pagination : $33-49$

ISSN : 2102-7188

\section{Référence électronique}

Claire Donnat-Aracil, « La dévotion aux saintes dans les Miracles de Nostre Dame de Gautier de Coinci une érotique du langage aux frontières de l'interdit », Questes [En ligne], 37 | 2018, mis en ligne le 01 février 2018, consulté le 20 avril 2019. URL : http://journals.openedition.org/questes/4456 ; DOI : $10.4000 /$ questes.4456 


\title{
La dévotion aux saintes dans les Miracles de Nostre Dame de Gautier de Coinci : une érotique du langage aux frontières de l'interdit
}

\author{
Claire DONNAT-ARACIL \\ Université Paris 3-Sorbonne Nouvelle
}

Moine et bénédictin à l'abbaye de Soissons puis au prieuré de Vicsur-Aisne, auteur d'un recueil de Miracles de Nostre Dame ${ }^{1}$ datant de la première moitié du XIII ${ }^{\mathrm{e}}$ siècle, Gautier de Coinci se situe dans la mouvance de la littérature mariale en langue vernaculaire qui se développe à partir du XII siècle, et sur laquelle s'est exercée la double influence de la lyrique des troubadours et des trouvères d'une part, et du roman courtois de l'autre. Au moment où la dévotion à Marie se propage, les auteurs qu'elle inspire développent un modèle littéraire marial fondé sur la reprise du motif de la dame courtoise, dont ils exploitent le potentiel religieux en le vidant - en principe - de l'érotisme inhérent au poème d'amour qui chante le désir interdit de la femme aimée ${ }^{2}$. C'est donc à travers la reprise et l'épuration d'un idéal qui est celui de la sexualité que se pense la dévotion à celle qui, en tant que vierge, est pourtant la plus étrangère au registre de la sexualité. Ce paradoxe se trouve particulièrement creusé dans l'œuvre de Gautier de Coinci, qui est loin de donner à lire une vision purement éthérée de la vie religieuse : la critique

\footnotetext{
${ }^{1}$ Gautier de Coinci, Les Miracles de Nostre Dame, éd. Victor Frédéric Koenig, Genève, Droz, 1955-1970, 4 tomes.

${ }^{2}$ Voir Michel Zink, Poésie et conversion au Moyen Âge, Paris, PUF, 2003 ; JeanLouis Benoit, «La dame courtoise et la littérature dans les Miracles de Nostre Dame de Gautier de Coinci », Le Moyen Âge, vol. CXVI, n 2, 2010, p. 367-384.
} 
a largement souligné sa propension à introduire dans ses récits des détails sulfureux ou à décrire avec minutie les troubles physiques qui accompagnent la tentation de luxure ${ }^{3}$. L'objet de cette étude est de réinterroger cette alternance entre sexualité et spiritualité en montrant que Gautier ne se contente pas d'allusions simplement ludiques au plaisir charnel, mais utilise le langage de l'érotisme pour traduire la force de la dévotion religieuse chez les personnages masculins particulièrement pieux ${ }^{4}$.

Une telle démarche n'est bien sûr pas entièrement neuve dans la tradition judéo-chrétienne, qui a pu voir dans le langage érotique une expression de l'union étroite de l'âme à Dieu. Le principal support à cette analogie a été le Cantique des Cantiques, auquel s'intéresse particulièrement le Moyen Âge occidental à partir du XII ${ }^{\mathrm{e}}$ siècle $^{5}$. Alors que la tradition exégétique cistercienne ${ }^{6}$ en propose uniquement une

\footnotetext{
${ }^{3}$ Voir sur ce point Marie-Christine Pouchelle, « Mots, fluides et vertiges : les fêtes orales de la mystique chez Gautier de Coinci », Annales. Économies, Sociétés, Civilisations, vol. 42, $\mathrm{n}^{\circ}$ 5, 1987, p. 1209-1230 ; Brian J. Levy, «Or escoutez une merveille! Parallel Paths : Gautier de Coinci and the fabliaux », dans Gautier de Coinci. Miracles, Music and Manuscripts, dir. Kathy M. Krause et Alison Stone, Turnhout, Brepols, 2006, p. 331-343.

${ }^{4}$ Nous nous concentrons ici sur les personnages masculins dans la mesure où la piété féminine demeure un thème très minoritaire chez Gautier de Coinci : sur l'intégralité des Miracles de Nostre Dame, seuls cinq contes prennent pour protagoniste une femme dont la sainteté demeure sans tache (I Mir. 19, I Mir. 29, II Mir. 9, II Mir. 24, II Mir. 27). Bien que les femmes entrées en religion parlent du Christ comme de leur « haut baron [et] haut espoz » (I Mir. 43, v. 315), cette relation sponsale n'est jamais chargée d'un caractère érotique, hormis dans le cas de II Ch. 10, Des nonains de Nostre Dame de Soissons, qui n'est pas un récit mais un sermon, et de II Mir. 9, De l'empeeris qui garda sa chasteé. Notre étude n'abordera toutefois pas la question de l'érotisme dans ce dernier conte, où la relation amoureuse au Christ s'insère dans un cadre particulier, celui d'une opposition entre le premier mariage de l'héroïne mariage humain - et ses épousailles avec le Christ après avoir été abandonnée de son époux terrestre. Les jeux de renversements autour du vocabulaire du désir et de l'amour dans ce récit pourraient à eux seuls faire l'objet d'une étude.

${ }^{5}$ Hildegard Elisabeth Keller, My Secret is mine. Studies on religion and eros in the German Middle Ages, Leuven, Sterling/Peeters, 2000, p. 14-19.

${ }^{6}$ Parmi les principaux commentaires cisterciens sur le Cantique, on peut citer Bernard de Clairvaux, Sermons sur le Cantique des cantiques, éd. Jean Leclerq, Henri Rochais
} 
lecture allégorique ${ }^{7}$, le sens littéral en est réactivé dans la littérature profane qui cite le Cantique pour conférer à l'amour et au désir humain qu'elle célèbre une dimension sacrée ${ }^{8}$. La littérature religieuse du Bas Moyen Âge réinvestira à son tour le sens littéral de ce texte à travers les expériences mystiques de femmes telles que Margery Kempe, pour qui l'union spirituelle au Christ prend la forme d'une relation sponsale charnelle et sensuelle ${ }^{9}$.

Le dialogue entre amour spirituel et amour charnel constitue donc une large problématique dans la conception chrétienne et médiévale de l'union mystique. Au sein de cette mosaïque, Gautier de Coinci présente la particularité de proposer un jeu d'alternance entre la compréhension littérale et la lecture purement spirituelle du thème cistercien du sponsus marianus, «l'époux de Marie ». Dans les différentes peintures de la dévotion portée à la sainte par les personnages masculins, on tentera d'observer que le langage de l'érotisme se déploie, autour des pratiques religieuses d'un protagoniste, en proportion de sa pureté de cœur.

Trois motifs ${ }^{10}$ permettront d'illustrer ce déploiement parallèle de la sainteté du héros et du langage érotique : la nuit de noces, où l'appel à la conversion du protagoniste attaché au monde conduit à opposer sensualité et sexualité ; l'enlacement de la sainte, où le langage érotique signale la

et Charles Hugh Talbot, trad. Paul Verdeven et Raffaele Fassetta, Paris, Cerf, 19962007, 5 tomes ; Guillaume de Saint-Thierry, Exposé sur le Cantique des cantiques, éd. Jean-Marie Déchanet, trad. Pierre Dumontier, Paris, Cerf, 2007 [1962].

${ }^{7}$ Voir sur ce point John W. Baldwin, Les Langages de l'amour dans la France de Philippe Auguste, Paris, Fayard, 1997, p. 252-256.

${ }^{8}$ John Brückmann et Jane Couchman, «Du Cantique des cantiques aux Carmina burana : amour sacré et amour érotique », dans L'Érotisme au Moyen Âge, dir. Bruno Roy, Montréal, L'Aurore, 1977, p. 37-50.

${ }^{9}$ Hildegard Keller, My Secret is mine, op. cit., p. 11-12.

${ }^{10}$ Le terme de «motif » sera employé ici pour désigner les « motifs narratifs » tels que les définit Jean-Pierre Martin par opposition aux «motifs rhétoriques ». Voir son article, «Les motifs dans la chanson de geste. Définition et utilisation », Cahiers de civilisation médiévale, vol. 30, n 120,1987 , p. 315-329. 
piété du dévot; le baiser au visage, où l'érotisme devient l'expression de l'Amour divin.

\section{La nuit de noces : la conversion du désir}

Le motif de la nuit de noces repose sur un schéma assez conventionnel : au cours de la nuit qui suit son mariage, un homme est appelé à choisir entre l'amour humain, charnel et peccamineux, et la pureté de l'amour divin. De ce topos caractéristique de la littérature religieuse, Gautier de Coinci fait cependant un usage intéressant en présentant la Vierge comme une rivale humaine et sensuelle de la femme légitime. C'est ce que l'on observe dans le conte I Mir. 21, De l'enfant qui mist l'anel ou doit l'ymage ${ }^{11}$, dans lequel un jeune homme, par jeu, passe un jour au doigt d'une statue de la Vierge l'anneau que lui a donné sa promise en gage d'amour ${ }^{12}$. Alors que la statue replie miraculeusement ses doigts pour empêcher le héros de récupérer l'anneau, signalant ainsi sa volonté de le voir entrer en religion, le clerc préfère épouser sa fiancée et s'attire la colère de Marie dont la jalousie se déclare au moment de la consommation du mariage :

Li clers, qui mise eut grant entente

En amer la gente pucele,

Qui mout estoit mignote et bele,

Mout desirra l'aller gesir

Por acomplir son grant desir.

Mais erranment qu'il vint ou lit,

Ne li sovint de nul delit,

Ainz s'en dormi tot sanz plus faire.

\footnotetext{
${ }^{11}$ Gautier de Coinci, Les Miracles de Nostre Dame, op. cit., t. II, p. 197-204.

12 Jean-Louis Benoit insiste sur le caractère transgressif que représente pour Gautier le choix même de raconter ce miracle, qui est à l'origine une légende païenne : "C'est Vénus qui veut se venger de l'infidélité de celui qui avait promis de s'unir à elle, avant de rompre ses vœux pour une femme. Il y a quelque risque à transposer, même par souci d'édification, une légende aussi sulfureuse dans le domaine chrétien (ce sont les devanciers de Gautier qui l'ont fait en latin) », art. cit., p. 374.
} 
La douce dame debonaire,

Qui douce est plus que mielz en ree,

Luez droit a lui s'est demostree.

Au clerc sambloit que Nostre Dame

Gisoit entre lui et sa fame ${ }^{13}$

La sexualité conjugale est ici vue à travers le prisme de références intertextuelles qui la cantonnent dans le domaine de l'échange purement physique ; on repère en effet des allusions à deux genres littéraires dont l'influence sur l'écriture de Gautier a été démontrée, le fabliau14 et le roman courtois ${ }^{15 .}$ La présence onirique de la Vierge dans le lit conjugal introduit le double motif du ménage à trois et du sommeil comme entrave à l'acte sexuel, qui peuvent évoquer des fabliaux érotiques comme Les Treces ${ }^{16}$ ou Le Sohait des Vez ${ }^{17}$. Le motif du rêve comme substitut de l'union charnelle peut également se trouver développé dans le roman courtois lorsqu'une héroïne cherche à éviter une union charnelle qu'elle considère illégitime $^{18 .}$ Enfin, le lexique employé peut rappeler l'univers du

${ }^{13}$ Gautier de Coinci, Les Miracles de Nostre Dame, op. cit., I Mir.21, v. 100-112.

${ }^{14}$ Brian J. Levy, «Or escoutez une merveille ! Parallel paths : Gautier de Coinci and the fabliaux », art. cit.

${ }^{15}$ John W. Baldwin, Les Langages de l'amour dans la France de Philippe Auguste, op. cit., p. 65-86. La référence simultanée au fabliau et à la littérature courtoise n'est pas un procédé étonnant pour Baldwin selon qui « la compétition très nette qui oppose [les fabliaux] aux romans vernaculaires oblige à lire les deux genres de concert », p. 83. Insistant sur la proximité de forme entre les deux genres, Baldwin affirme l'identité possible de public entre fabliaux et romans courtois : «Le fait que les fabliaux atteignaient aussi un public courtois est confirmé non seulement par les récits eux-mêmes, mais également par des témoignages émanant directement de la société aristocratique », p. 86.

${ }^{16}$ Les Treces, édité dans Fabliaux érotiques. Textes de jongleurs des $\mathrm{XII}^{\mathrm{e}}$ et XIII $^{\mathrm{e}}$ siècles, éd. Luciano Rossi et Richard Straub, Paris, Librairie générale française, 1993, p. 166-183.

${ }^{17}$ Le Sohait des Vez, édité dans Fabliaux érotiques, op. cit., p. 138-153. De retour après deux mois de voyage, un marchant rentre auprès de sa femme qui lui prépare un festin largement arrosé de vin dans l'espoir de lui donner envie de faire l'amour: «Mais de ce ne fist pas savoir / que del vin l'a si empressé / que li vins l'i a confessé, / et, qant vint au cochier el lit, / qu'il oblia l'autre delit » (v. 40-44, p. 140).

${ }^{18}$ Le don à l'époux ou à l'amant non agréé d'un sort ou d'un breuvage lui faisant rêver qu'il s'unit charnellement à la femme qu'il désire est en effet une ruse utilisée par 
fabliau en nommant l'acte sexuel en termes non métaphoriques («gesir », v. 102 ; «acomplir son grant desir », v. 103 ; «a sa fame n'a geü », v. 140 et «touche a sa fame », v. 166). Ces termes demeurent certes très éloignés du langage cru et de l'inventivité érotique des fabliaux, mais s'en rapprochent en nommant franchement la réalité sexuelle sans faire usage des euphémismes propres au langage romanesque ${ }^{19}$. Ainsi désignée en termes physiologiques, la sexualité se distingue au final de l'érotisme, puisque le conteur n'aspire pas à éveiller chez le lecteur une forme de désir $^{20}$. L'intertextualité contribue dès lors à mettre à distance, voire à tourner en dérision une sexualité conjugale dans laquelle la femme légitime demeure d'ailleurs intégralement passive et se montre presque absente du texte.

En regard de l'union conjugale qui est donc pensée comme une sexualité dépourvue de sensualité, le récit donne à Marie le rôle de l'amante en la désignant comme «amie» («N'autre amie ne devoit avoir », v. 79), terme que la Vierge assume elle-même dans son discours («Loial amie en moi eüssez», v. 124) où elle s'oppose à la femme légitime : «tu por ta chaitive fame / M'as renoïe et deguerpie » (v. 156157). Ces indices invitent à placer la dévotion à la Vierge du côté de l'union des cœurs, à rebours de la dimension sociale inhérente au mariage, ce que confirme la description de l'entrée en religion du héros comme une fuite précipitée, nocturne et clandestine :

Dou lit saut sus, plus n'i demeure,

Si l'espira la bele dame

Fénice pour échapper à l'empereur dans le Cligès de Chrétien de Troyes, ou par la dame du lac pour obtenir ses pouvoirs de Merlin dans le Lancelot en prose.

${ }^{19}$ Sur le refus du roman de désigner clairement l'acte sexuel, voir John Baldwin, Les Langages de l'amour dans la France de Philippe Auguste, op. cit., p. 218.

${ }^{20}$ Sur la lecture comme moyen d'éveiller chez le lecteur le désir sexuel, voir Evelyn Birge Vitz, «La lecture érotique au Moyen Âge et la performance du roman», Poétique, vol. 137, n 1, p. 35-51. 
Qu'ainc n'esvilla home ne fame,

Ainz s'en fui en hermitage

Et prist habit de moniage.

La servi Dieu toute sa vie

Et ma dame sainte Marie.

$\mathrm{Ne}$ volt au siecle remanoir :

Avec s'amie ala manoir,

Cui il avoit por amor mi

L'anel ou doit con vrais amis ${ }^{21}$.

La peinture de l'entrée en religion comme un départ nocturne, certes assez fréquente dans la littérature religieuse vernaculaire ${ }^{22}$, débouche au vers 180 sur la représentation de Marie en amie terrestre et en compagne quotidienne. En assimilant l'entrée en religion à un mariage d'amour (v. 182-184), le conteur calque ce récit de vocation sur le modèle de romans courtois comme le Cligès ${ }^{23}$.

La consécration à la Vierge se présente donc pour l'instant sous la forme de noces amoureuses mais dégagées de la pratique sexuelle propre au mariage terrestre. En renonçant à l'amour charnel, le personnage opère une conversion du désir qui rattache la sensualité, non plus à la sexualité, mais à l'amour marial. D'autres motifs mettant en scène des personnages plus avancés en sainteté s'acheminent toutefois vers une réconciliation entre sexualité et spiritualité.

\footnotetext{
${ }^{21}$ Gautier de Coinci, Les Miracles de Nostre Dame, op.cit., I Mir. 21, v. 172-182.

${ }^{22}$ Paul Bretel, «La nuit de l'ermite », dans Littérature et édification au Moyen Âge. «Mult est diverse ma matyre », Paris, Champion, 2012, p. 71-92.

${ }^{23}$ Les derniers vers du Cligès de Chrétien de Troyes insistent également sur le double lien qui unit Fénice et le héros, à la fois amants et époux : «De s'amie a faite sa femme, / Mes il l'apele amie et dame, / Car por ce ne pert ele mie / Que il ne l'aint come s'amie, / Et ele lui tot autresi / Com amie doit son amie » (v. 6671-6676). Sur l'influence de cette œuvre sur l'écriture de Gautier de Coinci, voir Olivier Collet, Glossaire et index critiques des $œ$ uvres d'attribution certaine de Gautier de Coinci, Genève, Droz, 2000.
} 


\section{L'enlacement de la sainte : le langage érotique, expression de piété}

Le motif de l'enlacement de la sainte donne lieu au déploiement d'une plus grande sensualité pour évoquer la dévotion d'hommes d'Église accomplissant parfaitement leur charge de pasteurs et serviteurs de Dieu. Gautier de Coinci ne développe pas ce motif autour de Marie, mais autour de sainte Léocadie, à laquelle il consacre deux récits placés en écho à l'ouverture et à la clôture du premier livre des Miracles. Léocadie y est à deux reprises l'objet d'une dévotion exprimée par l'étreinte du protagoniste, Ildephonse dans le premier récit, et, dans le second, Gautier de Coinci lui-même qui se met en scène comme prieur de Vic-sur-Aisne.

L'histoire d'Ildephonse est narrée dans le conte D'un archevesque qui fu a Tholete ${ }^{24}$. Réputé pour sa sainteté, Ildephonse porte une dévotion particulière à Léocadie qui, lors d'une cérémonie publique, sort miraculeusement de son tombeau à la prière de l'évêque :

[...] Ainc n'i ot prince, duc ne conte, Tant fust hardis, abbés n'evesques

Qui l'atouchast fors l'archevesques

[...]

Li soutilz clers, li bien apris,

Que Sainz Esperites espris

Et enbrasé eut de sa flame,

Enbracier s'amie et sa dame

Hardiement et tost $\mathrm{osa}^{25}$.

[...]

L'archevesques assez plora

Quequ'entre ses bras demora

La sainte virge Leochade ${ }^{26}$.

$[\ldots]$

\footnotetext{
${ }^{24}$ Gautier de Coinci, Les Miracles de Nostre Dame, op.cit., t. II, I Mir. 11, D ’un archevesque qui fu a Tholete, p. 5-103.

${ }^{25}$ Ibid., v. 112-121.

${ }^{26}$ Ibid., v. 133-135.
} 
Li sainz hom plainz dou Saint Espir

Mout ra geté parfont souspir

Quant voit que la sainte pucele,

Qui tant est symple et tant est bele,

Dont a tel joie et tel solas,

Li reschaspe d'entre ses bras

Et en sa fosse s'en retrait ${ }^{27}$.

Le texte met l'accent sur le geste inouï posé par l'évêque, en le mentionnant à quatre reprises : «l'atouchast» (v. 114), «enbracier s'amie » (v. 120), « entre ses bras demora » (v. 134), «d'entre ses bras » (v. 150). La sensualité de son initiative est signalée par l'insistance sur l'audace du personnage («tant fust hardis », v. 113, « hardiement et tost osa », v. 121), ainsi que par la connotation érotique du vers 149 («sont a tel joie et tel solas »), puisque la présence de la jeune femme dans les bras de l'évêque contribue à donner à «joie » et « solas » le sens qu'ils ont fréquemment dans la littérature courtoise, où ils constituent des euphémismes désignant l'acte amoureux ${ }^{28}$. L'érotisme du geste posé coïncide pourtant avec l'affirmation de sa valeur sainte, puisque le héros agit «espris / Et enbrasé [...] de [la] flame» du Saint-Esprit» (v. 118119). La paronomase «embrasié / embracier» (v. 119-120) invite bien à lire ce geste sensuel comme procédant d'un amour spirituel, signe du lien privilégié qui unit Ildephonse à la sainte et à Dieu.

À l'autre bout du premier livre des Miracles, Gautier de Coinci se fait lui-même le protagoniste du miracle 44, Comment sainte Leochade fu perdue $^{29}$. Ce récit se réfère en partie à l'histoire d'Ildephonse, que l'auteur

\footnotetext{
${ }^{27}$ Ibid., v. 145-151.

${ }^{28}$ John W. Baldwin, Les Langages de l'amour dans la France de Philippe Auguste, op. cit., p. 218 : «les romanciers refusent uniformément de nommer l'acte sexuel de façon explicite mais préfèrent le suggérer par des mots dénotant le plaisir. Joie, delit, deduit et solas $[\ldots]$ sont les termes couramment employés pour désigner les rapports sexuels ».

${ }^{29}$ Gautier de Coinci, Les Miracles de Nostre Dame, op.cit., t. III, I Mir. 44, p. 214 248.
} 
présente à plusieurs titres comme son modèle et son double fictionnel : comme Gautier, Ildephonse était homme d'Église et poète, comme lui il a rédigé un livre à la louange de Marie. Surtout, Gautier tient à se présenter comme l'héritier et le rival de l'archevêque de Tolède dans sa dévotion privilégiée à Léocadie, dont le prieuré de Vic-sur-Aisne, qu'il dirigeait, possédait des reliques. Dans ce dernier miracle, le conteur met en scène le vol de ces reliques par des hommes dont le but est de conserver la châsse en or. Lorsqu'il apprend leur recouvrement, Gautier se rend impatiemment au-devant du convoi ramenant les reliques sans le reliquaire :

Quant vins a li, si me doloie

Faillue ja m'estoit l'alainne.

Ains n'embraça Paris Helainne

Si doucement con je fis li.

Mon duel eu tot enseveli

Tout maintenant que je la tins ${ }^{30}$.

$[\ldots]$

Haute pucele de haut pris,

De roial sanc nee et estraite,

Trop laidement t'avoient traite

Ta chemise li malfaiteur ${ }^{31}$.

Dans cette seconde scène d'enlacement, le conteur élude les éléments qui pourraient rappeler qu'il ne serre dans ses bras qu'un morceau de reliques ${ }^{32}$, terme effacé dans les lignes qui précèdent au profit de « corps» («le cors la sainte damoysele », v. 187) et de « pucele» (v. 192), et dans l'extrait cité au profit du pronom féminin («vins a li », « je la tins »). Une transposition similaire se retrouve dans la mention de

\footnotetext{
${ }^{30}$ Ibid., v. 296-301.

${ }^{31}$ Ibid., v. 324-327.

${ }^{32}$ Gautier de Coinci, Les Miracles de Nostre Dame, op.cit., dans I Mir. 11, D’un archevesque qui fu a Tholete, le conteur narre le transfert de l'Espagne à la France des reliques de Léocadie, constituées par ce qu'Ildephonse a prélevé de la sainte, à l'aide d'un couteau, au moment où elle lui est apparue : il « en trancha ce qu'en peut avoir » (v. 173) et « en un vaisel d'or et d'argent / Tout en apert, voiant la gent, / Mist ce qu'il avoit de s'amie » (v. 185-187).
} 
la châsse emportée par les voleurs, qui devient une « chemise » qu'on lui $\mathrm{a}$ «traite » (v. 326-327). Ces éléments concourent à une humanisation des reliques de la sainte ${ }^{33}$, dont le vol est tiré, par la mention d'un vêtement aussi intime que la chemise ${ }^{34}$, du côté du viol. En transformant ainsi la scène du retour processionnel des reliques en des retrouvailles avec la femme aimée, Gautier de Coinci tente donc de s'élever au niveau de l'intimité qu'a partagée Ildephonse avec Léocadie, et même de la dépasser: alors que la sainte ressuscitée apparaissait hiératique et insensible aux embrassements de l'évêque malgré leur quadruple évocation, Gautier parvient en deux vers à transformer son geste dévotionnel en un témoignage d'amour passionnel et partagé, en se comparant à Pâris embrassant Hélène (v. 298). La proximité avec la sainte se traduit, non plus par un simple vocabulaire amoureux, mais par la référence à une relation adultère.

Gautier met ainsi en place un procédé singulier dans le paysage de la littérature monastique de son temps : alors que les écrits théologiques des XII ${ }^{e}$ et XIII ${ }^{e}$ siècles n'abordent un texte biblique aussi sensuel que le Cantique qu'à condition de le spiritualiser par une lecture allégorique, Gautier adopte la démarche inverse et sensualise le geste commun de

\footnotetext{
${ }^{33}$ Voir Pierre-André Sigal, L'Homme et le miracle dans la France médiévale ( $\mathrm{XI}^{\mathrm{e}}-$ XII ${ }^{\mathrm{e}}$ siècle), Paris, Cerf, 1985, p. 35-36; Henri Platelle, «Guibert de Nogent et le De pignoribus sanctorum. Richesse et limites d'une critique médiévale des reliques », dans Les Reliques. Objets, cultes, symboles, dir. Edina Bozoky et Anne-Marie Helvétius, Turnhout, Brepols, 1999, p. 109-121. Si, selon Sigal, l'assimilation des reliques à la personne même du saint est courante au Moyen Âge, et fondée sur les écrits des Pères, Platelle montre les objections avancées au XII ${ }^{\mathrm{e}}$ siècle par Guibert de Nogent, pour qui seul le Christ est spirituellement présent dans notre monde par l'Eucharistie.

${ }^{34}$ Sur la valeur érotique et féminine de l'évocation de la chemise, voir Romaine WolfBonvin, «Un vêtement sans l'être : la chemise », dans Le Nu et le vêtu au Moyen Âge $\left(\mathrm{XII}^{\mathrm{e}}-\mathrm{XIII}{ }^{\mathrm{e}}\right.$ siècles), dir. Chantal Connochie-Bourgne, Aix-en-Provence, Publications de l’Université de Provence, 2001, p. 383-394.
} 
dévotion que représente l'embrassement d'une relique ${ }^{35}$. La force de l'expérience spirituelle ne peut s'exprimer qu'à travers une érotisation du langage qui, par la référence à Pâris et Hélène, conduit la dévotion religieuse dans le domaine interdit de la relation adultère et funeste.

On peut toutefois distinguer un niveau encore plus profond de dévotion qui atteint à l'union mystique, où la sensualité du langage traduit un geste véritablement érotique.

\section{Le baiser à la Vierge : l'érotisme comme langage de l'union mystique}

Le motif du baiser vient réconcilier érotisme des mots et érotisme du geste, puisqu'il s'agit à la fois d'un baiser réellement donné, et exalté par la langue poétique sur le modèle, convoqué par le poète, du Cantique des cantiques.

Dans le conte 31 du premier recueil, Dou soucretain que Nostre Dame visita $^{36}$, un moine supplie chaque nuit la Vierge de le laisser un jour la voir avec les yeux de l'âme. Une nuit, elle lui apparaît en songe, et le sacristain lui demande l'autorisation d'embrasser ses pieds, ce à quoi Marie répond par la négative :

Je ne veil mie,

Biaus doz amis, que ja atouche

A mes piez ta sainte de bouche,

Qui tant de fois m'a saluee ;

Mais en ma face coloree,

Biaus douz amis, me siet et plaist

Que ta bele bouche me baist. »

La douce dame glorieuse,

La debonaire, la piteuse,

\footnotetext{
${ }^{35}$ Embrasser les reliques fait en effet partie des gestes de dévotion communément pratiqués par les pèlerins dans les sanctuaires. Voir Pierre-André Sigal, L'Homme et le miracle dans la France médiévale, op. cit.

${ }^{36}$ Gautier de Coinci, Les Miracles de Nostre Dame, op.cit., t. III, I Mir. 31, p. 11-22.
} 
Doucement s'est vers lui baissie ;

Cil l'a tout en plorant baisie

En sa face, qu'a plus rovente

Que n'est florete en florie ente.

As piez li est plorant cheüs,

Si est de joie rapeüs

Et si est plains de grant leece

Que bien li samble que tristrece,

Destorbier, painne et malaige

N'ara ja mais en son aaige . $^{37}$.

Le conteur tend ici au lyrisme par l'usage de phrases longues, de nombreux enjambements qui créent un rythme ample et par le vocabulaire antithétique de la joie et de la douleur. Ce lyrisme de l'écriture, joint à l'insistance sur l'intériorité du personnage et le sentiment de plénitude débordante qui l'habite, contribue à faire oublier que le baiser n'a pas été donné à la Vierge sur la bouche, mais seulement sur sa «face», de sorte que la sensualité de l'écriture vient ainsi dépasser l'érotisme inhérent au geste du baiser d'amour.

Cet érotisme du langage demeure indissociable de la dimension sacrée conférée à la description de la relation sensuelle, à travers la présence du Cantique des cantiques qui se laisse deviner en intertexte ${ }^{38}$. Outre la reprise d'un vocabulaire amoureux qui peut tenir aussi bien du

\footnotetext{
${ }^{37}$ Ibid., v. 162-180.

${ }^{38}$ L'identification de Marie à la fiancée du Cantique des cantiques se répand à partir du XII ${ }^{\mathrm{e}}$ siècle, où le poème est lu comme le récit des fiançailles du Christ et de la Vierge (voir John W. Baldwin, Les Langages de l'amour dans la France de Philippe Auguste, op. cit., p. 253). On peut noter toutefois l'étonnante répartition des rôles opérée ici : si Gautier de Coinci fait prononcer à Marie les mots de la fiancée («Que ta bele bouche me baist »), c'est elle qui donne au moine le baiser divin, endossant ainsi le rôle masculin qui est le propre de la divinité dans la compréhension du Cantique des Cantiques par les Pères de l'Église et les théologiens (voir sur ce point Hildegard Keller, My Secret is mine, op. cit., p. 21-51). Keller souligne le contraste entre la nécessité de penser l'âme comme féminine dans son union au Dieu masculin, et la difficulté pour un homme de se penser devant Dieu comme femme. Dans les scènes racontées par Gautier, cette aporie se résout partiellement puisqu'il y a une altérité sexuelle au départ, même si la répartition des rôles Époux/épouse est instable au cours du récit.
} 
texte biblique que de la poésie des trouvères ${ }^{39}$, certains passages renvoient assez précisément au Cantique et à son exégèse. La parole de la Vierge au vers 168, «Que ta belle bouche me baist », peut ainsi se lire comme une paraphrase du verset d'ouverture du poème biblique, "Qu'il me baise d'un baiser de sa bouche ». Bien plus, la reprise de ce premier verset du Cantique après la demande du moine d'embrasser les pieds de Marie fait écho à l'exégèse cistercienne : si le passage du baiser des pieds au baiser de la bouche constitue une progression hiérarchique familière de la pensée médiévale ${ }^{40}$, le contexte invite plutôt à voir, dans cet extrait, une réminiscence du schéma des trois baisers de l'âme au Christ décrit par Bernard de Clairvaux dans les huit premiers sermons qu'il consacre au commentaire du Cantique ${ }^{41}$. Méditant le début in medias res de ce livre qu'il interprète comme un dialogue de l'âme-épouse avec le divin Époux, Bernard développe l'idée que l'audace inhérente au fait de demander à Dieu «un baiser de sa bouche» ne peut être que l'ultime étape d'un parcours spirituel éludé par le texte, qu'il métaphorise par trois baisers : l'âme a d'abord «insisté avec maintes prières et bien des larmes pour mériter au moins de toucher ses pieds ${ }^{42} »$, obtenant «le pardon des offenses par le baiser des pieds », puis réclamé le « baiser des mains » qui

\footnotetext{
${ }^{39}$ À titre d'exemple, l'apparition de la Vierge «plus clere que solaus / A mïedi quant est plus haus» (v. 83-84) peut évoquer le Cantique, VI, 9 ( «Quae est ista quae progreditur quasi aurora consurgens, [...] electa ut sol...») aussi bien que des vers d'un troubadour comme Bernart de Ventadorn («Ara no veil luzir solehl... »).

${ }^{40}$ Voir Yannick Carré, Le Baiser sur la bouche au Moyen Âge. Rites, symboles, mentalités, à travers les textes et les images, $\mathrm{XI}^{\mathrm{e}}-\mathrm{XV}^{\mathrm{e}}$ siècles, Paris, Le Léopard d'Or, 1993, p. 27-30.

${ }^{41}$ Bernard de Clairvaux, Sermons sur le Cantique, op. cit., t. I.

${ }^{42}$ Ibid., p. 194-197. Dans le sermon IX, Bernard glose le dialogue entre l'épouse et les compagnons de l'époux en imaginant ces derniers interrogeant la fiancée errante sur les raisons de sa tristesse : "nonne ut saltem merereris tangere pedes, multis precibus et fletibus institisti ? [...] Obtento eo simulque accepta in osculo pedum indulgentia de offensis ».
} 
lui a conféré la force de ne plus pécher ${ }^{43}$, avant d'oser demander le baiser de la bouche :

Postremo cum ista multis precibus et lacrimis obtinemus, tunc demum audemus forsitan ad ipsum os gloriae caput attollere, pavens et tremens dico, non solum speculandum, sed etiam osculandum [...]. Et nunc quid restat, o bone Domine, nisi ut iam in plenitudine lucis, in fervore spiritus, ad oris quoque osculum dignanter admittens, adimpleas me laetitia cum vultu tuo ${ }^{44}$ ?

Les précautions rhétoriques employées ici par le prédicateur («audemus forsitan », «pavens et tremens dico ») signalent le caractère exceptionnel d'une telle union. A fortiori, la demande formulée chez Gautier de Coinci par la Vierge elle-même, et non par le dévot, traduit à la fois l'humilité du personnage, qui se sent indigne de poser un geste placé par Bernard en bas de l'échelle de l'élévation spirituelle, et l'amour de prédilection que Marie lui porte.

Alors que Bernard de Clairvaux proposait donc une interprétation allégorique de la sensualité du Cantique, Gautier semble ici reprendre l'exégèse qu'a élaborée le cistercien sous forme de métaphore filée, pour en vivifier le sens littéral jamais activé par Bernard. Le motif du baiser à la Vierge apparaît ainsi comme un signe d'élection spirituelle très particulier, et ce dès le choix de l'intertexte : la référence au Cantique des cantiques, dont les théologiens du XII ${ }^{\mathrm{e}}$ siècle s'accordent à considérer la

\footnotetext{
${ }^{43}$ Ibid. p. 196-197 : «[...] ita ut in osculo manus adepta sis virtutes non paucas et non parvas ».

${ }^{44}$ Ibid., sermon 3, p. 109-111 : «Enfin, quand nous avons obtenu ces faveurs par bien des prières et des larmes, alors seulement nous osons, peut-être, lever la tête vers la bouche glorieuse elle-même, non pas simplement pour la contempler, mais, je le dis tremblant de crainte, pour y poser un baiser. [...] Et maintenant, Seigneur de bonté, que te reste-t-il à faire, sinon me combler de joie par ton visage, en daignant me permettre aussi le baiser de la bouche, dans la plénitude de la lumière, dans la ferveur de l'esprit? » (nous soulignons).
} 
lecture dangereuse pour ceux qui ne sont pas déjà entrés profondément dans la connaissance et l'amour de Dieu, est en elle-même un indice de la supériorité de l'âme du protagoniste ${ }^{45}$. En proposant de ce texte et de son exégèse une interprétation littérale et sensuelle, Gautier de Coinci fait du contact charnel accordé au protagoniste le gage de la pureté de son cœur.

Au terme de cette typologie non exhaustive, on peut donc associer à chaque motif étudié un positionnement face au lien entre érotisme et spiritualité : le héros du miracle 21 jouait le rôle du « commençant $^{46}$ » dans la vie religieuse. Son difficile renoncement aux choses du monde était signalé par la référence aux littératures profanes (fabliau et roman courtois) dans le cadre desquelles la dévotion à Marie se définissait comme une relation entre amants, dégagée cependant de l'érotisme. Gautier et Ildephonse pourraient représenter les «progressants »: l'érotisme autour du motif de l'enlacement était présent, mais se développait à proportion inverse de la sensualité véritable du geste posé.

\footnotetext{
${ }^{45}$ Voir John W. Baldwin, Les Langages de l'amour dans la France de Philippe Auguste, op. cit., p. 253. L'auteur y rend compte du raisonnement de Pierre le Chantre selon qui le roi Salomon a composé trois œuvres: «les Proverbes, qui traitent de problèmes de morale ou d'éthique à l'usage des débutants, l'Ecclésiaste, qui traite de la nature physique des choses à l'intention de ceux qui sont plus avancés, et enfin le Cantique des cantiques, qui traite des figures divines de la théologie pour ceux qui sont mûrs et parfaits, ceux-là seuls qui atteignent à l'amour et à la paix de Dieu. Il est particulièrement important, ajoute-t-il, que ceux qui lisent cette dernière œuvre aient une connaissance parfaite et une foi solide, car le texte risquerait d'éveiller la concupiscence chez un esprit lubrique et immature. » Bernard de Clairvaux obéit à la même logique dans son premier sermon sur le Cantique, en réservant la lecture de ce poème à ceux qui ont déjà tiré pleinement bénéfice des deux autres œuvres de Salomon, en apprenant à dompter l'amour du monde par la lecture de l'Ecclésiaste, et l'amour de soi par la lecture du livre des Proverbes : «Autrement, sans avoir d'abord dompté la chair et l'avoir assujettie à l'esprit par les exercices de la discipline, sans avoir méprisé et rejeté l'apparat et l'attirail du monde, c'est indignement que des gens impurs prétendent à cette lecture sainte » (Bernard de Clairvaux, Sermons sur le Cantique, op. cit., p. 64-65).

${ }^{46} \mathrm{La}$ distinction proposée entre «commençants », «progressants » et «parfaits » renvoie à la typologie mise en place par Guillaume de Saint-Thierry dans sa Lettre aux frères du Mont-Dieu, éd. et trad. Jean Dechanet, Paris, Cerf, 1985.
} 
Le protagoniste du miracle 31 a atteint quant à lui la «perfection spirituelle»: l'union mystique à Marie lui est accordée sans qu'il la demande, et le langage érotique épouse ici la sensualité du baiser donné. La progression des personnages en sainteté se traduit ainsi par la disparition du besoin de purifier ou d'allégoriser l'interdit monastique que constitue l'échange charnel et sensuel.

À observer ce procédé, on peut penser que ce lien entre sensualité et spiritualité traduit une conception de la dévotion quelque peu aux marges du dogme, ce dont Gautier de Coinci semble lui-même conscient puisqu'il cherche toujours à mettre à distance la scène d'union dévotionnelle : soit par le biais du rêve dans les miracles 21 et 31, soit en transposant l'objet de la dévotion amoureuse, qui n'est plus Marie mais une sainte de second ordre, Léocadie. Gautier développe donc des stratégies pour atténuer le potentiel séditieux de ces récits. Ce potentiel séditieux n'est toutefois pas à lire comme une volonté, de la part de l'auteur, de s'affranchir de l'institution ecclésiale. Ces différents motifs seraient plutôt à relier à la rhétorique de l'indicible omniprésente lorsque le conteur cherche à décrire la grandeur de Marie, qui est douce, nous ditil, «plus que ne puet langue retraire ${ }^{47}$ ». Face aux limites de la langue religieuse et littéraire pour exprimer la violence de l'expérience spirituelle, l'érotisme poétique serait le seul recours : il ne resterait au moine que le langage de l'amour interdit pour dire la rencontre inénarrable avec le divin Amour.

\footnotetext{
${ }^{47}$ Gautier de Coinci, Les Miracles de Nostre Dame, op.cit., II Mir. 28, v. 378.
} 
